\title{
Spontaneous Polarization and Orientational Dynamics of Polar Rod-like Molecules in Host/Guest Materials
}

\author{
M. Wübbenhorst, J. van Turnhout, G. Klap \\ Department of Polymer Materials and Engineering \\ Faculty of Applied Sciences \\ Delft University of Technology \\ Delft, The Netherlands \\ J. C. Jansen \\ Department of Applied Organic Chemistry and Catalysis \\ Faculty of Applied Sciences \\ Delft University of Technology \\ Delft The Netherlands
}

\begin{abstract}
A. Quintel and J. Hulliger
Department of Chemistry and Biochemistry

University of Bern Berne, Switzerland
\end{abstract}

\begin{abstract}
The polar domain structure and dynamics of host/guest materials which include polar rodlike molecules were investigated by pyroelectric and dielectric techniques. Using scanning pyroelectric microscopy (SPEM), spatially resolved information on a $\mu \mathrm{m}$ scale was obtained of the 3-D domain structure of the polarization caused by an acentric host lattice and/or the polar arrangement of dipolar guest molecules. Two classes of pyroelectric host/guest materials were examined with SPEM: (1) an inorganic host (zeolite $\left.\mathrm{AlPO}_{4}-5\right)$ which was loaded with $p$ nitroaniline (PNA) guest molecules by an adsorption process, and (2) an organic channel-type inclusion material, which establishes its macroscopic polarity by cocrystallization of perhydrotriphenylene (PHTP) with the polar guest 1-(4-nitrophenyl)piperazine) (NPP). Despite great differences in the chemical composition and morphology, both types of host/guest systems revealed $180^{\circ}$ macro-domains, of which the particular shape and sign of the polarization were in accordance with models about crystal growth and/or directed adsorption of polar molecules. In order to assess the molecular dynamics of the included guest molecules, frequency dependent local pyroelectric measurement, based on SPEM, and broad-band dielectric relaxation spectroscopy were performed. Whereas guest molecules included in PHTP channels show no rotational dynamics, we found several relaxation processes in PNA-loaded $\mathrm{AlPO}_{4}-5$ crystals, which were assigned to local and cooperative relaxation modes of molecular chains of the hydrogen-bonded PNA guest molecules.
\end{abstract}

\section{INTRODUCTION}

C HANNEL-TYPE inclusion compounds and zeolite based host/ guest systems are promising materials for crystal engineering of stable and highly efficient 2 nd order nonlinear optically active and pyroelectric materials. Their common feature is the ability to establish a noncentrosymmetric arrangement of hyperpolarizable molecules in structural voids, either by co-crystallization of guest and host molecules (inclusion crystals $c f$. Figure 1(a) to (c) or due to directional adsorption of guest molecules in zeolite channels (cf. Figures 1(d) to (f) and 2). The molecular mechanisms for polar growth in inclusion compounds [1], as well as for directed adsorption of $p$-nitroaniline (PNA) molecules in $\mathrm{AlPO}_{4}-5$ [2], have been investigated in the last few years, leading to 
the understanding of the key mechanisms $[3,4]$.

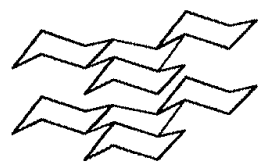

a<smiles>O=[N+]([O-])c1ccc(N2CCNCC2)cc1</smiles>
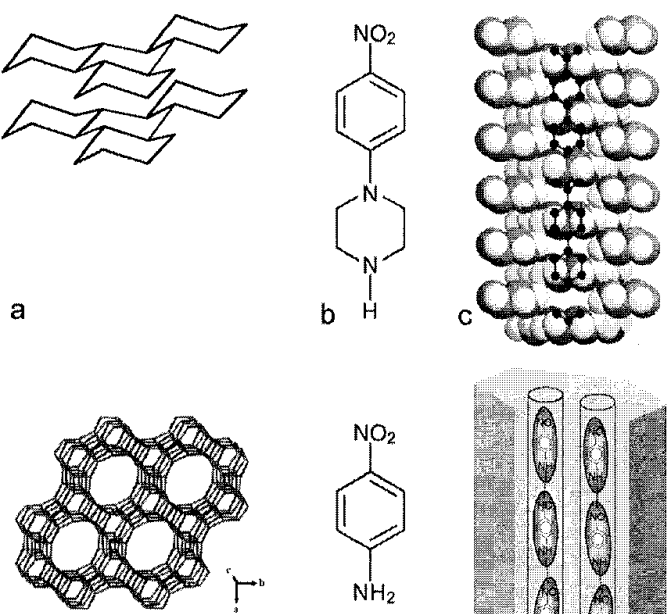

d<smiles>Nc1ccc([N+](=O)[O-])cc1</smiles>

e

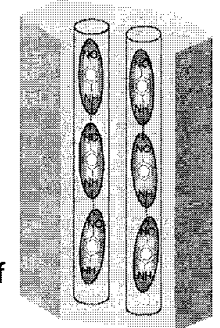

Figure 1. Schematic representation of two different polar host/guest structures and their building units. above: (a) stack of 2 PHTP molecules, (b) guest compound NPP and (c) lattice model of a PHTP-NPP inclusion crystal; below: (d) view on the $\mathrm{AlPO}_{4}-5$ framework along the channel axis, (e) structure of PNA and (f) illustration of the channel system of $\mathrm{AlPO}_{4}-5$ filled with PNA guest molecules.

In order to study the polarization direction and possible domain structures of the macroscopic polarization in polar materials, we have applied a new scanning probe technique based on local pyroelectric measurements (SPEM). After a brief description of the experimental technique, we will discuss the pyroelectric information obtained for polar inclusion crystals and PNA-loaded zeolites, which yielded details on the growth process of the (guest) host crystal or peculiarities of the adsorption behavior of $\mathrm{AlPO}_{4}-5$.

Not much is known about the molecular dynamics of polar host/ guest materials, except that full reorientation of the included polar guest molecules by $180^{\circ}$ flips is practically impossible for steric reasons. The very absence of such a relaxation process of the guest molecules in both zeolite-based and PHTP-based host/guest systems makes them attractive as polar materials. This can be rationalized easily for PHTP inclusion crystals, where rod-like guest molecules like 1-(4nitrophenyl)piperazine) (NPP) are tightly packed in the PHTP channels and are stabilized by van der Waals forces and e.g. guest-guest hydrogen bonds. In contrast, the formation of polar order in the nano-porous zeolite $\mathrm{AlPO}_{4}-5$ during the filling process requires sufficient molecular mobility for diffusion of PNA guest molecules over macroscopic distances along rigid tubes of diameter $\sim 0.73 \mathrm{~nm}$. Polarized Fourier transform infrared (FTIR) measurements have shown that restricted torsional fluctuations of the PNA molecules from the $c$-axis (channel axis) exist and that the mean fluctuation angle and the degree of $\mathrm{H}$ bonding between PNA molecules are temperature dependent even at room temperature [5].

Recently, dielectric spectroscopy on $\mathrm{AlPO}_{4}-5 / \mathrm{PNA}$ crystals has revealed a strong relaxation process that was assigned to cooperative motions of the one-dimensional PNA-chains [6], since the relaxation time

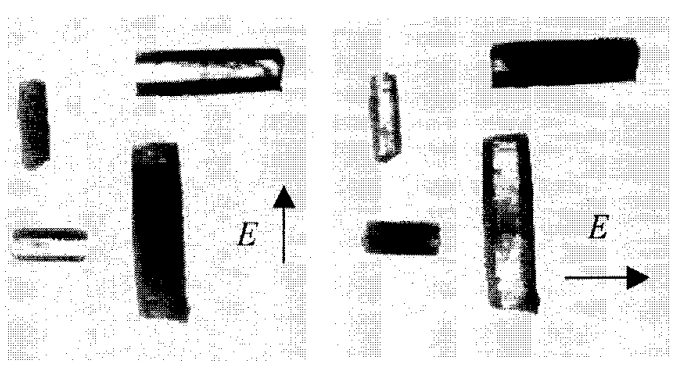

Figure 2. Microscopic view of differently oriented $\mathrm{AlPO}_{4}-5 / \mathrm{PNA}$ crystals in polarized light, revealing the molecular orientation of the PNA molecules parallel to the crystal axis.

followed a Vogel-Fulcher-Tammann (VFT) behavior. In the present paper we describe results from (dynamic) pyroelectric experiments on the same materials, which allow the study of individual crystals. A comparison between dielectric and pyroelectric relaxation times will be made.

\section{MATERIALS AND SAMPLE PREPARATION}

\subsection{ZEOLITE SAMPLES}

Large $\mathrm{AlPO}_{4}-5$ crystals were synthesized in Teflon ${ }^{\mathrm{TM}}$ lined autoclaves with triethylamine as a template. To remove the organic template, the crystals were calcined at $700^{\circ} \mathrm{C}$ for $48 \mathrm{~h}$. The removal of the template was verified with FTIR measurements, the crystal structure was checked by X-ray diffraction. Freshly calcined crystals were filled with PNA from the vapor phase at $155^{\circ} \mathrm{C}$ for $\sim 4 \mathrm{~h}$. This procedure resulted in an average degree of filling with PNA molecules of $\sim 5 \%$ wt as verified by FTIR and thermogravimetric analysis.

\subsection{PREPARATION OF PHTP INCLUSION COMPOUNDS}

In this study, inclusion crystals based on perhydrotriphenylene (PHTP) and the donor/acceptor (DA) disubstituted chromophore NPP were investigated. Needle-shaped crystals of 1 to $3 \mathrm{~mm}$ in length and 0.2 to $0.5 \mathrm{~mm}$ in thickness were obtained by crystallization from solution, resulting in crystals with a nearly hexagonal cross section. The needle axis corresponds to the channel axis. Further details concerning the crystal growth and the sample preparation are described in [7].

\section{EXPERIMENTAL SETUP}

\subsection{SCANNING PYROELECTRIC MICROSCOPY}

A schematic representation of the SPEM equipment is shown in Figure 3. The sinusoidally modulated beam of a laser diode is focused by use of the objective of a conventional microscope onto the blackened surface of a pyroelectric crystal. The charges generated are collected on large electrodes that are connected to a current amplifier (Keithley 428 , conversion factor $10^{8}$ to $10^{10} \mathrm{~V} / \mathrm{A}$ ). A digital lock-in amplifier (Stanford Research 850 ) provides the sinusoidal modulation voltage and phase sensitive analysis of the response. In order to perform the scanning of the sample surface the whole sample/electrode system is 
mounted on a motorised $X-Y$-displacement unit. More experimental details are given in [8].

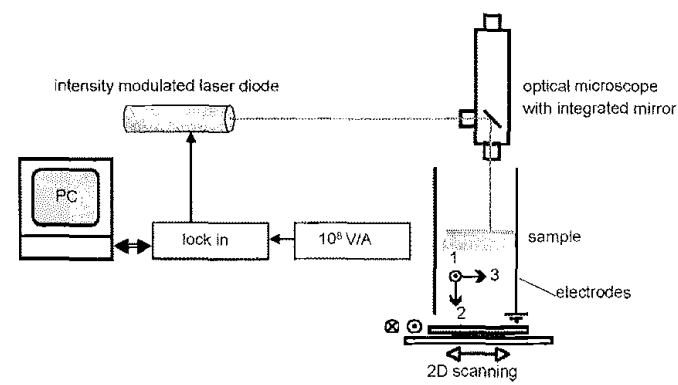

Figure 3. Experimental setup of SPEM.

To ensure a high resolution of the pyroelectric measurements, two requirements must be fulfilled

1. high optical resolution, i.e. small spot size of the laser beam $(d \approx 5 \mu \mathrm{m})$, and

2. short thermal diffusion length $\mu=\sqrt{K / \pi f}$ ( $K$ is the thermal diffusivity, $f$ the modulation frequency) with $\mu \leqslant d$.

To match these conditions for polymers and organic crystals $(K \approx$ $10^{-7} \mathrm{~m}^{2} / \mathrm{s}$ ) frequencies in the $\mathrm{kHz}$ range are necessary (case a in Figure 4). However, if low frequencies are used - which cause semispherical heat profiles (case b in Figure 4) - one can probe the pyroelectric activity in deeper regions of the sample. In this case, the lateral resolution reduces correspondingly with increasing $\mu$.

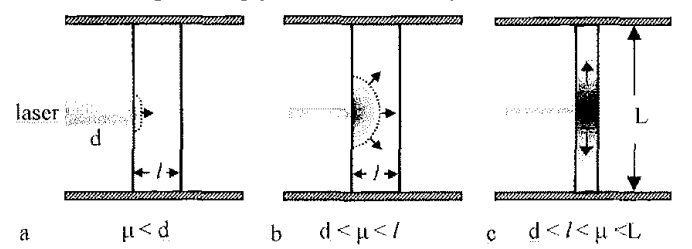

Figure 4. Thermal profiles for different thermal diffusion lengths in rodlike samples between oversized electrodes. The situation on the far left (a) ensures the highest possible thermal resolution.

\subsection{PYROELECTRIC SPECTROSCOPY}

In order to perform temperature and frequency dependent pyroelectric experiments on individual $\mathrm{AlPO}_{4}-5 / \mathrm{PNA}$ crystals, the SPEM setup was combined with a hot/cool stage (Linkam THM 600), which was modified by a sample holder for accurate crystal positioning. A laser spot of $\sim 50 \mu \mathrm{m}$ in diameter was adjusted such that the crystal was heated close to the free end of the crystal. According to [9] this resulted in probing the pyroelectric activity in a volume of the crystal, the size of which depends on the modulation frequency. Consequently, the domain contributing to the pyroelectric response varied from the irradiated crystal's half (at high frequencies, $f>100 \mathrm{~Hz}$ ) to the whole crystal (at low frequencies). Since both halves are pyroelectric with opposite sign, low frequencies result in a decrease of the pyroelectric response due to the influence of the bipolar polarization profile. For this reason the practically usable frequencies were limited to range from $2 \mathrm{~Hz}$ to $2 \mathrm{kHz}$.
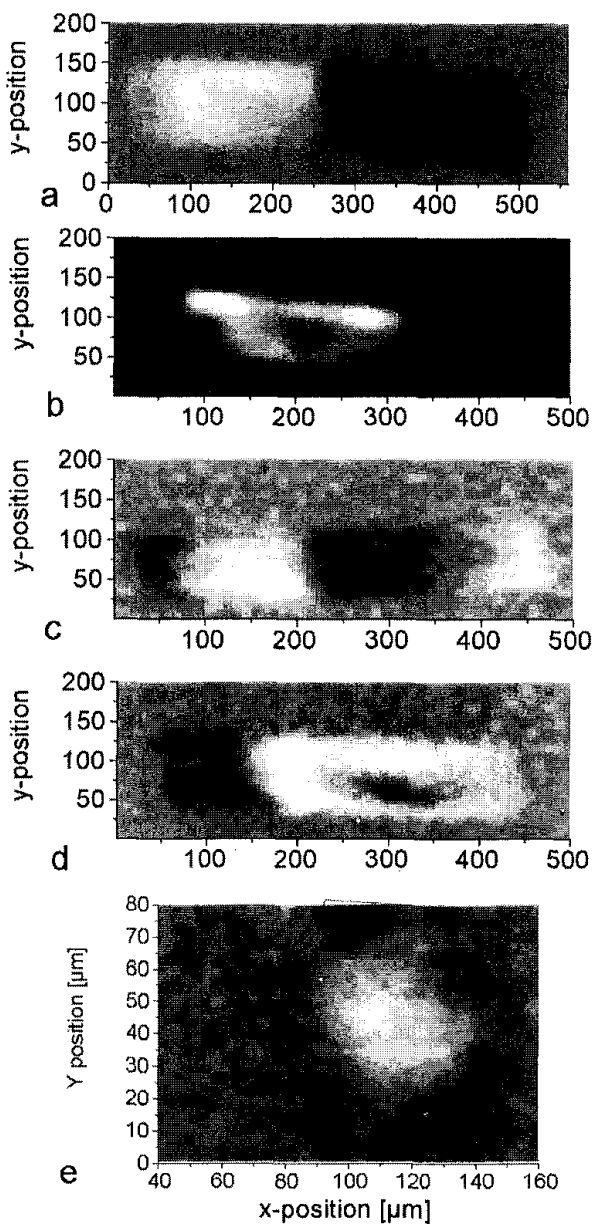

Figure 5. Pyroelectric images obtained on various $\mathrm{AlPO}_{4}-5$ crystals: (a) complete (twinned) empty crystal ( -1 to $1 \mathrm{pA} f=2000 \mathrm{~Hz}, \Delta x$, $\Delta y=10 \mu \mathrm{m}$ ), (b) single domain crystal grown on a substrate (black $=0$, white $=$ positive current), (c) abnormally grown crystal showing 3 polarization reversals, (d) asymmetrically grown and incompletely calcined crystal; the black area within the white part probably indicates residuals from the organic template, (e) cross section of an abnormally grown crystal with core exhibiting a polarization opposite to that of the outer regions. In all Figures (except (b)) the pyroelectric current is shown on a gray scale, reaching from black (negative current) via half-gray (zero) to white (positive current).

\subsection{DIELECTRIC SPECTROSCOPY}

For dielectric measurements, PNA loaded $\mathrm{AIPO}_{4}-5$ crystals were put between circular brass electrodes of $20 \mathrm{~mm}$ in diameter, resulting in a moderately packed layer ( $\sim 40 \%$ vol packing density) with a thickness of $\sim 85 \mu \mathrm{m}$ of preferentially planar oriented crystals. Preparation took place either in vacuum or under flowing gaseous nitrogen. Dielectric experiments were performed using a combination of three dielectric measurement systems covering a broad frequency range from $10^{-2} \mathrm{~Hz}$ to $10^{9} \mathrm{~Hz}$ :

1. a frequency response analyzer (Schlumberger 1260) equipped with a custom made dielectric interface (developed by TNO) for frequencies between $10^{-2}$ and $10^{3} \mathrm{~Hz}$, 
2. a Hewlett-Packard $4284 \mathrm{~A}$ precision LCR-meter for frequencies between $10^{3}$ and $10^{6} \mathrm{~Hz}$, and

3. a Hewlett-Packard rf-analyzer HP4291 A for the frequency range from $10^{6}$ to $1.8 \times 10^{9} \mathrm{~Hz}$.

The sample was placed in a nitrogen cryostat (Novocontrol), the temperature of which was controlled with a stability of better than $\pm 50 \mathrm{mK}$.

\section{POLAR DOMAINS IN ZEOLITES FILLED WITH DIFFERENT GUEST MOLECULES}

The SPEM measurements on empty crystals of aluminophosphate $\mathrm{AlPO}_{4}-5$ with channel-like nanopores always revealed a polarization reversal in the middle part of these crystals (Figure 5(a)) [10]. This behavior can be ascribed to the crystallization process, in which the crystals grow freely from a seed into opposite directions with one particular absolute configuration. However, a single domain crystal is formed when the crystal is grown from a substrate (Figure 5(b)).

In some cases, especially after fast crystallization, deviating crystal shapes and complex domain structures were found. This can be caused, for example, by the presence of more defects, intergrowth of crystals in the early stage, dendritic growth, or secondary nucleation. A few examples are shown in Figures 5(c) to (e). Because the material grows with the polarization direction always the same as the growth direction, the SPEM images contain information about the growth history of the sample. In other words, the domain structure after growth represents the frozen-in evolution of the growth process. Figure 5(e) shows a crosssection of a crystal in which the core of the crystal has an opposite polarization compared to that of the surface region. The SEM images of samples from different stages in the crystallization process confirm that these parts actually grew in an opposite direction.

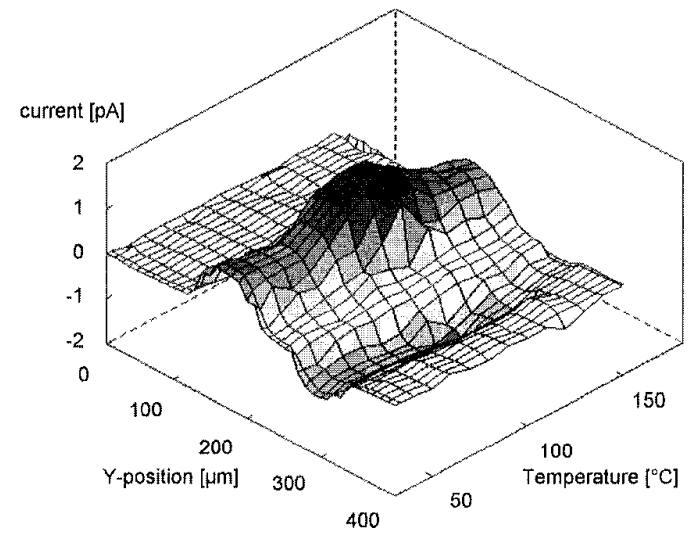

Figure 6. Temperature dependent line scans along the $c$-axis of an $\mathrm{AlPO}_{4}-5$ crystal loaded with PNA.

So far only the pyroelectric properties of empty $\mathrm{AlPO}_{4}-5$ crystals were considered. It has been shown earlier $[5,10]$, that loading of $\mathrm{AlPO}_{4}-5$ with PNA results in much stronger pyroelectric materials, which exhibit the same polarization reversal as the empty host crystal ( $c$ f. line scan, Figure 6). For comparison, the temperature dependent pyroelectric coefficient of (calcined) $\mathrm{AlPO}_{4}-5$ and $\mathrm{AlPO}_{4}-5 / \mathrm{PNA}$ is given in the same graph (Figure 7). An interesting detail is the drop of the pyroelectric activity for empty $\mathrm{AlPO}_{4}-5$ at $\sim 50{ }^{\circ} \mathrm{C}$ due to the pyroelectric/ paraelectric phase transition.

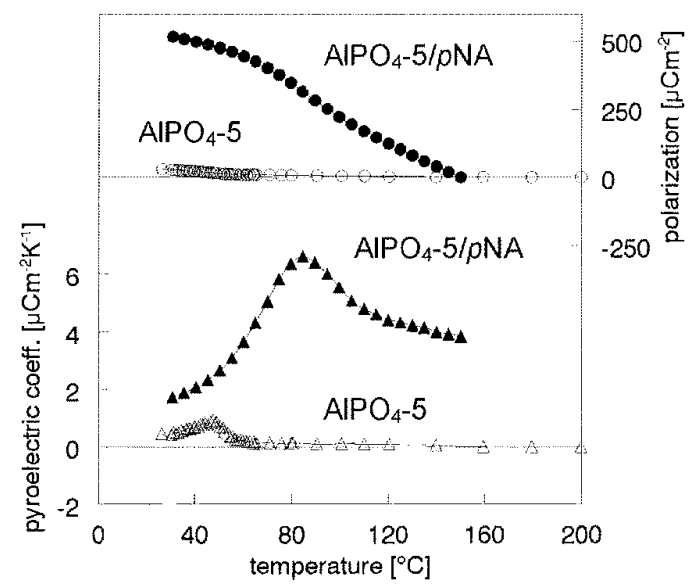

Figure 7. (Bottom) pyroelectric coefficient $p(T)$ (absolute value) of an empty (open symbols) and a PNA loaded $\mathrm{AlPO}_{4}-5$ single crystal (filled symbols) as a function of temperature. (Top) relative spontaneous polarization $P$ obtained by integration of $p(T)$. Note that $P$ was set to zero at the highest temperature of the integration interval.

The molecular origin of the strong pyroelectric effect in $\mathrm{AlPO}_{4}$ 5/PNA is the formation of aligned dipole chains with opposite directions in the two halves of the crystal. It has been shown in many experiments that the PNA molecules always orient preferentially with respect to the polar axis of the $\mathrm{AlPO}_{4}-5$ host lattice. An explanation is an orientation selective adsorption mechanism driven by specific molecular interactions of the different substituents of the polar molecules with the capping surfaces of the $\mathrm{AlPO}_{4}-5$ crystals [2].

\section{POLAR DOMAIN STRUCTURE IN PHTP BASED INCLUSION COMPOUNDS}

Another successful application of SPEM was the characterization of the 3-dimensional polarization distribution in a polar perhydrotriphenylene PHTP based inclusion compound. Although the PHTP host lattice and most of the pure guest crystal structures themselves are centrosymmetric, the inclusion crystals appeared to be polar for most of the tested polar guest molecules (e.g. NPP, 4-iodo-4'-nitrobiphenyl (INBP)) [11].

A first important result from SPEM imaging on PHTP-NPP inclusion crystals was the observation of macrodomains featuring opposite polarizations $[7,12]$. Recent SPEM experiments of the crystal core region, utilizing an improved spatial resolution $(\sim 10 \mu \mathrm{m})$, showed an hourglass shaped domain structure, which was predicted by a growth model based on Markov's theory of stochastic processes. Figure 8 gives an example of a successively thinned PHTP-NPP crystal, yielding the lateral polar domain structure at three different depths. In particular, the third scan (Figure 8(c)), which refers to a layer $100 \mu \mathrm{m}$ beneath the original surface, shows conical structures pointing to the suggested seed region. Whereas the domain structure is rather complicated in the center of the 

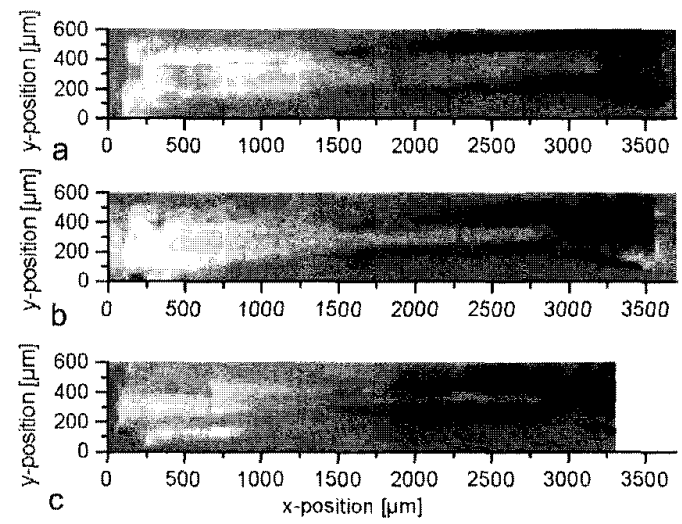

Figure 8. SPEM images of a PHTP-NPP crystal at the surface (a) and after removing a layer of $60 \mu \mathrm{m}(\mathrm{b})$ and $100 \mu \mathrm{m}(\mathrm{c})$. Gray scale: black $=$ $-0.3 \mathrm{pA}$, white $=+0.3 \mathrm{pA}$. Step size: $20 \mu \mathrm{m}, f=415 \mathrm{~Hz}(\mu \approx 10 \mu \mathrm{m})$.

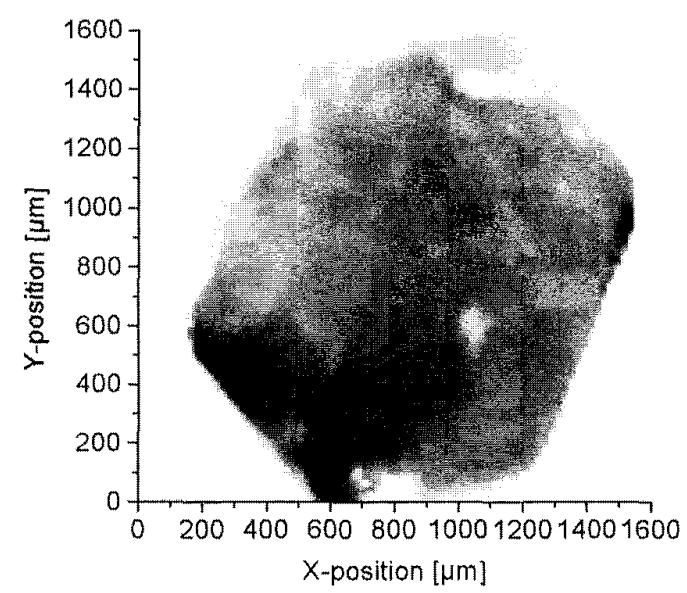

Figure 9. SPEM image of one of the two capping faces of a large PHTPNPP crystal revealing an almost homogeneous pyroelectric activity. Step size: $20 \mu \mathrm{m}, f=415 \mathrm{~Hz}$. The gray scale ranges from white $=0 \mathrm{pA}$ to black $=1.8 \mathrm{pA}$.

crystal, the polarization becomes almost homogeneous near the capping faces of the crystal (Figures 8(a) to (c)).

In order to scan a capping face we have replaced one of the metal sample electrodes by a transparent (indium tinoxide (ITO) coated glass) electrode. The result of a scan with the laser beam parallel to the crystal's needle axis is presented in Figure 9 and confirms the suggested unipolar state at the end of the growth process.

\section{DYNAMICS OF pNA GUESTS IN ALPO $4-5$}

\subsection{DIELECTRIC RELAXATIONS}

Dielectric experiments on $\mathrm{AlPO}_{4}$-5/PNA powder samples were performed isothermally at temperatures increasing and decreasing stepwise $(\Delta T=2.5 \mathrm{~K})$. After a first heating run to $195^{\circ} \mathrm{C}$, during which residual absorbed water was removed, the samples were slowly cooled to $-150^{\circ} \mathrm{C}$. Subsequent repeated heating and cooling showed a highly reproducible relaxation behavior that hardly depends on the direction of the temperature variation. Typical spectra are given in Figure 10. Three well-separated relaxations show up:

1. the fastest and weakest ( $\beta$ relaxation) becomes visible only at $T>50^{\circ} \mathrm{C}$, its peak maximum shifts only a little with temperature,

2. a strong asymmetric $\alpha$ relaxation, and

3. a slow process (II), the peak frequency of which is $>4$ decades lower than $f_{\alpha}$ and which depends slightly on the thermal history of the sample.

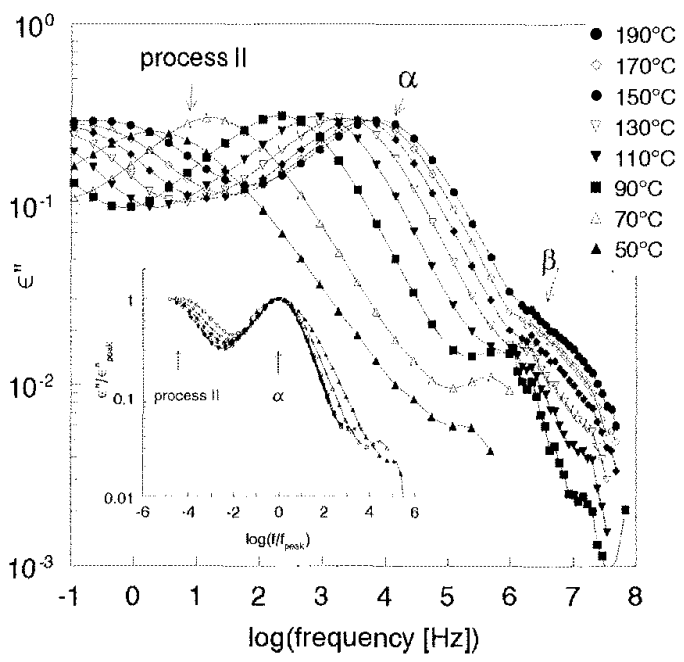

Figure 10. Dielectric loss spectra of $\mathrm{AlPO}_{4}-5 / \mathrm{PNA}$ (with $\sim 5 \% \mathrm{PNA}$ ) at eight selected temperatures, measured during cooling from $195^{\circ} \mathrm{C}$. Inset: The same spectra normalized to peak frequency and peak maximum (master plot). $190^{\circ} \mathrm{C}, \diamond 170^{\circ}, 150^{\circ} \mathrm{C}, \nabla 130^{\circ} \mathrm{C}, \nabla 110^{\circ} \mathrm{C}, 9^{\circ} \mathrm{C}, \Delta 70^{\circ} \mathrm{C}$, $\triangle 50^{\circ} \mathrm{C}$.

For a quantitative analysis we fitted the two strong relaxation processes in the loss spectra with Havriliak-Negami (HN) functions according to Equation (1)

$$
\varepsilon^{\prime \prime}=-\sum_{k=1}^{2} \mathfrak{I}\left\{\frac{\Delta \varepsilon_{k}}{\left[1+\left(i \omega \tau_{k}\right)^{a_{k}}\right] b_{k}}\right\}
$$

where $\Delta \varepsilon_{k}$ and $\tau_{k}$ denote the relaxation strength and the mean relaxation time of the $k$ th process, and $\mathfrak{I}$ indicates the imaginary part. The two peak shape parameters $a_{k}$ and $b_{k}$, which determine the slope of the low frequency tail $m=a$ and the high frequency tail $n(-a b)$, are related to the underlying distribution in relaxation times. The peak relaxation frequencies of both relaxation processes $v s$. the inverse temperature are presented in Figure 11.

The most striking result is the behavior of $f_{\alpha}$ (or $\left.\tau_{\alpha}=\left(2 \pi f_{\alpha}\right)^{-1}\right)$, the temperature dependence of which at high $\left(75<T<160^{\circ} \mathrm{C}\right)$ temperatures can be described quite well by a VFT Equation (2) with the parameters $\log \left(\tau_{\infty}\right)[\mathrm{s}]=-5.8, E_{V}=3.4 \mathrm{~kJ} / \mathrm{mol}$ and $T_{V}=296 \mathrm{~K}$.

$$
\tau(T)=\tau_{\infty} \exp \left[\frac{E_{V}}{R\left(T-T_{V}\right)}\right]
$$

Such a VFT-behavior is typical for a dynamic glass transition and indicates cooperative rearrangements of the participating (PNA) molecules on a temperature dependent cooperativity length scale $\xi(T)$. At $T \sim 72^{\circ} \mathrm{C}$ the relaxation time $\tau_{\alpha}$ departs markedly from the VFT law, indicating a change in the cooperative dynamics; this usually defines 


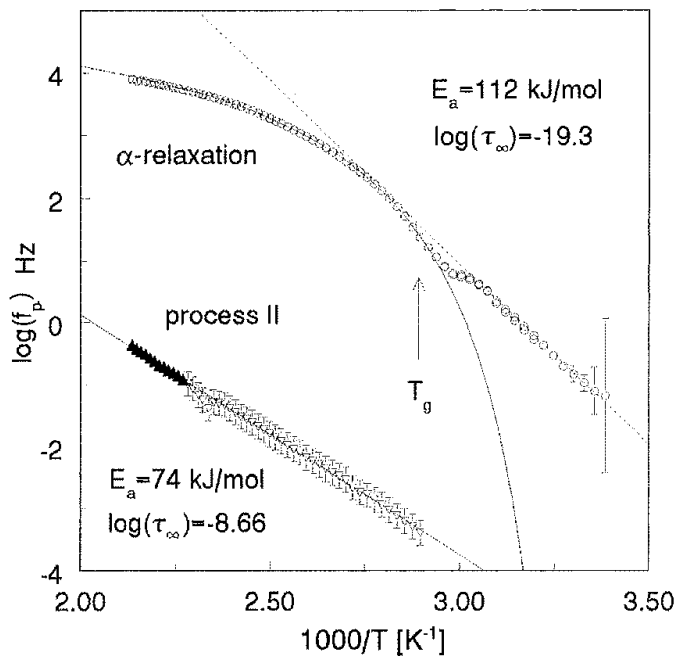

Figure 11. Arrhenius plots of the peak relaxation frequency $f_{p}$ for $\mathrm{AlPO}_{4}-5 / \mathrm{PNA} \%$. $1 /$ temperature. The relaxation peak data of process II at low temperatures $(\triangle)$ were extrapolated by a constrained $\mathrm{HN}$-fit, assuming temperature independent values for $\Delta \varepsilon_{2}$ and $a_{2}$ in Equation (1). The two fit curves of the (upper) $\alpha$ process were obtained by fitting the high temperature data (solid line) and the low temperature data (dashed line) with the VFT and the Arrhenius-equation separately.

the glass transition temperature $T_{g}$. Further lowering of the temperature clearly reveals an Arrheniuslike behavior of $\tau_{\alpha}$ which can be analyzed conveniently, since the relaxation rate at $T_{g}$ is unusually high $\left(f_{T_{g}}=24 \mathrm{~Hz}\right)$. The Arrhenius parameters of the $\alpha$ relaxation as well as those of process II are given in Figure 11.

The observation of liquid-like dynamics in one-dimensional molecular chains of PNA is far from self-evident. Recently, Huwe et al. [13,14] studied the relaxation behavior of ethylene glycol (EG) confined to zeolite host systems with different pore sizes. These authors found that $\mathrm{co}^{-}$ operative (liquid-like) dynamics already exists in ensembles of as small as 6 EG molecules as manifested by a VFT behavior of the relaxation time. By contrast, zeolitic hosts with smaller pores or cages, resulting in arrangements of EG molecules with a lower average number of neighboring molecules $(<6)$, showed a drastically increased relaxation rate which followed an Arrhenius law. Since in $\mathrm{AlPO}_{4}-5$ each individual PNA molecule can have only two directly interacting neighbors, other long-range PNA-PNA interactions along and across the zeolite channels must be taken into account to explain the cooperative dynamics.

Leike and Marlow [15] have shown by model calculations that PNA molecules adsorbed in the straight channels of $\mathrm{AlPO}_{4}-5$ might even undergo a first order phase transition when both intra- and (long-range) interchannel dipole-dipole interactions are considered. The existence of a VFT type behavior, therefore, supports the idea that both intrachannel ( $\mathrm{H}$ bonding, van der Waals and electrostatic forces) and interchannel interactions (only electrostatic interactions) evolve to 3-dimensional rather than one-dimensional interactions of the PNA molecular ensemble.

The second peculiarity of the dielectric $\alpha$ process, the pronounced change in its temperature dependence from VFT to Arrhenius behavior, has been discussed in detail in [6], in terms of the cooperativity length $\xi$ of the $\alpha$ process. In this view, the change from VFT to Arrhenius behavior marks the temperature below which $\xi$ probes the size of PNA molecular ensembles.

\subsection{PYROELECTRIC SPECTROSCOPY AND COMPARISON WITH DIELECTRIC RESULTS}

The manifestation of a molecular relaxation process in the pyroelectric response became already clear by temperature dependent pyroelec-

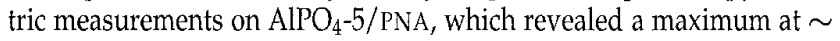
$85^{\circ} \mathrm{C}$ and $f=1 \mathrm{kHz}$, see Figures 6 and 7 . Systematic pyroelectric measurements using different frequencies, i.e. pyroelectric spectroscopy experiments, were performed using a similar temperature schedule as for the dielectric experiments. Again, the samples first were dried at $185^{\circ} \mathrm{C}$ and then measured during slow cooling to $25^{\circ} \mathrm{C}$. A typical result is given in Figure 12, which shows the absolute value of the pyroelectric current as function of temperature and frequency. All curves clearly reveal a peak at high frequencies that shifts toward lower temperatures at lower frequencies. The similar behavior of the high-frequency curves at low temperatures $\left(T<60^{\circ} \mathrm{C}\right)$ as well as the additional local maximum $T \sim 55^{\circ} \mathrm{C}$ for the low-frequency curves can be attributed to the pyroelectric $\rightarrow$ paraelectric transition of the $\mathrm{AlPO}_{4}-5$ lattice itself [10].

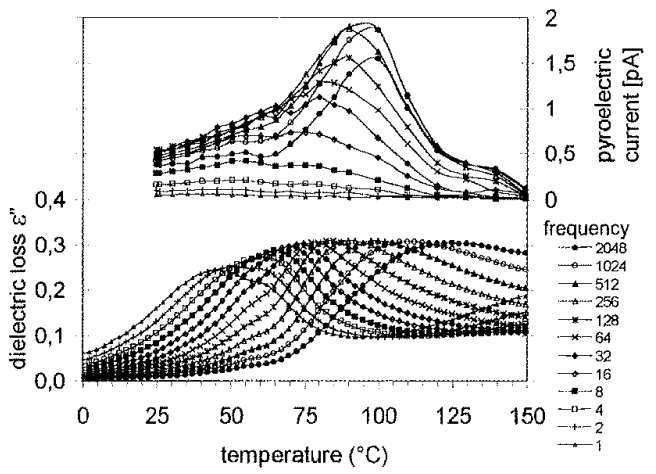

Figure 12. (Top) Pyroelectric current of an $\mathrm{AlPO}_{4}-5 / \mathrm{PNA}$ single crystal as function of temperature and frequency; (bottom) dielectric loss of an $\mathrm{AlPO}_{4}-5 /$ PNA powder sample.

For comparison, the pyroelectric relaxation data are presented together with the dielectric relaxation loss maxima in Figure 13. On first sight, there is a fair agreement between the pyroelectric and dielectric relaxation peak frequencies, which clearly implies a common molecular. relaxation mechanism. Nevertheless, the pyroelectric relaxation seems to be significantly slower than the dielectric $\alpha$ process. This prompted us to re-analyze the dielectric loss data by using two symmetric $\mathrm{HN}$ functions for the $\alpha$ peak instead of one asymmetric HN function. A good reason was the particular asymmetric shape of the $\alpha$ process $(m<n)$, which is very unlikely for cooperative $\alpha$ relaxations. As a result, the dielectric $\alpha$ process could be decomposed in two well separated relaxation processes, one close to the overall peak maxima, the other more than one decade slower than the fast relaxation mode.

Given the fact that two dielectric and one prominent pyroelectric relaxation modes were encounterd, one should visualize the possible cooperative relaxation modes for an $\mathrm{H}$-bonded PNA chain segment. Two 


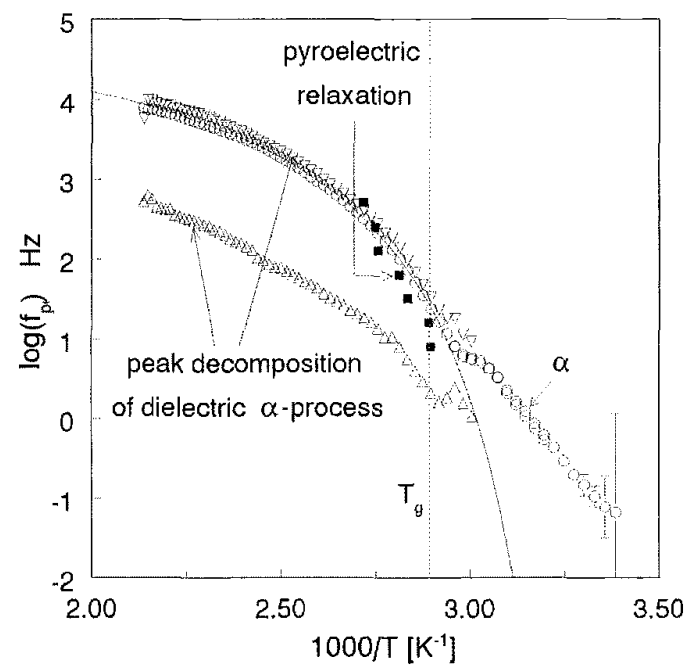

Figure 13. Arrhenius plots of the dielectric and pyroelectric $\alpha$ peak relaxation frequency $f_{p}:(0)$ dielectric $\alpha$ relaxation replotted from Figure 11, $(\mathbb{\Delta})$ pyroelectric relaxation obtained from Figure $12,(\triangle, \nabla)$ individual processes of the dielectric $\alpha$ peak determined by peak decomposition.
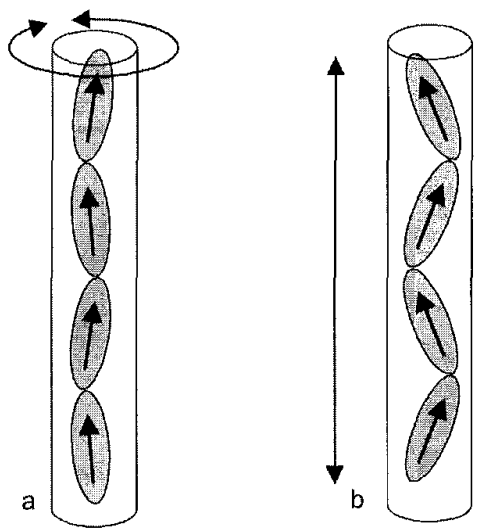

Figure 14. Illustration of two possible cooperative relaxation modes for a H-bonded PNA chain.

likely relaxation mechanisms are suggested in Figure 14(a) cooperative rotation of the whole chain segment around its axis (\|l to the tube axis), and (b) cooperative chain expansion and contraction, which is probably the slower process. Although individual PNA molecules posses only a longitudinal dipole moment, it is evident, that a PNA chain as a whole might have both a longitudinal and a transversal net dipole moment. Consequently both motional processes suggested in Figure 14 should be detectable in a dielectric experiment. By contrast, the pyroelectric experiment probes fluctuations of the permanent polarization caused by small temperature perturbations $(p=d P / d T)$.

Since the permanent polarization possesses only a longitudinal component (the polarization direction), the relaxation process according to Figure 14(b) is the only mechanism visible as pyroelectric relaxation. In this sense the pyroelectric relaxation frequencies given in Figure 13 are rather too high than too low. However, the discrepancies are still within the experimental uncertainties, e.g. caused by pronounced orientation and depolarization effects due to the very different sample geometries in the dielectric and pyroelectric experiments.

\section{CONCLUSIONS}

Scanning pyroelectric microscopy has proven to be a powerful tool for the spatially resolved pyroelectric characterization of host/guest materials. Two types of host/guest materials were investigated,

1. the zeolite $\mathrm{AlPO}_{4}-5 / \mathrm{PNA}$, and

2. an organic channel-type PHTP-NPP inclusion crystal, both reveal $180^{\circ}$ macro-domains of the polarization.

Besides simple polarization reversals, a few examples for more complicated domain shapes were discussed in terms of models for the crystal growth and/or the filling process $\left(\mathrm{AIPO}_{4}-5 / \mathrm{PNA}\right)$.

In addition to the static polarization domain structure, the molecular dynamics of PNA molecules confined in the nano-channels of $\mathrm{AlPO}_{4}-5$ was elucidated by broad-band dielectric relaxation spectroscopy and pyroelectric spectroscopy, based on SPEM. These complementary techniques revealed independently the 'liquid-like' cooperative dynamics of PNA chain fragments, which is indicated by the VFT temperature dependence of the corresponding $\alpha$ relaxation. In particular, two coupled dielectric relaxation modes and one pyroelectric relaxation process were found, the existence of which is in fair agreement with a proposed cooperative relaxation mechanism for molecular chains of hydrogenbonded PNA guest molecules.

\section{REFERENCES}

[1] J. Hulliger, P. Rogin, A. Quintel, P. Rechsteiner, O. König, and M. Wübbenhorst, "The crystallization of polar, channel-type inclusion compounds: property-directed supramolecular synthesis", Adv. Mater, Vol. 9, pp. 677-680, 1997.

[2] G. J. Klap, M. Wübbenhorst, J. C. Jansen, H. van Koningsveld, H. van Bekkum, and J. van Turnhout, "Polar Growth and Directional Adsorption of Large $\mathrm{AlPO}_{4}-5$ Crystals Determined by Scanning Pyroelectric Microscopy", Chem. Mater., Vol. 11, pp. 3497-3503, 1999.

[3] J. Hulliger, "Orientational disorder at growing surfaces of molecular crystals: general comments on polarity formation and on secondary defects", Z. Kristallogr., Vol. 214, pp. 9-13,1999.

[4] J.Hulliger, S. W. Roth, A. Quintel and H. Bebie, "Polarity of Organic Supramolecular Materials: a Tunable Crystal Property", J. Solid State Chem., 2000, in press.

[5] F. Marlow, M. Wübbenhorst, and J. Caro, "Pyroelectric Effects on Molecular Sieve Crystals Loaded with Dipole Molecules", J. Phys. Chem., Vol. 98, pp. 12315-12319, 1994.

[6] M. Wübbenhorst, G. J. Klap, J. C. Jansen, H. van Bekkum, and J. van Turnhout, "Glass transition of one-dimensional molecular chains of $p$-nitroaniline confined in $\mathrm{AlPO}_{4}-5$ nanopores revealed by dielectric spectroscopy", J. Chem. Phys., Vol. 111, pp. 5637-5640, 1999.

[7] A. Quintel, J. Hulliger, and M. Wübbenhorst, "Analysis of the polarization distribution in a polar perhydrotriphenylene inclusion compound by scanning pyroelectric microscopy", J. Phys. Chem. B, Vol. 102, pp. 4277-4283, 1998.

[8] M. Wübbenhorst, J. van Turnhout, A. Quintel, and J. Hulliger, "Spatially Resolved Heat Conduction in Polar Perhydrotriphenylene Inclusion Compounds Studied With Thermal Wave Techniques"', J. Appl. Phys., submitted, 2000.

[9] M. Wübbenhorst, G. J. Klap, J. van Turnhout, A. Quintel, and J. Hulliger, "Characterization of Dipole Alignment in Host/Guest Materials with Scanning Pyroelectric Microscopy", IEEE Proceedings of 10th Intern, Symp, on Electrets (ISE 10), Delphi, Greece, $22-24$ September 1999, pp. 469-472.

[10] G. J. Klap, S. M. van Klooster, M. Wübbenhorst, J. C. Jansen, H. van Bekkum, and J. van Turnhout, "Polarization Reversal in $\mathrm{AlPO}_{4}-5$ Crystals Containing Polar or Nonpolar Organic Molecules: A Scanning Pyroelectric Microscopy (SPEM) Study", J. Phys. Chem. B, Vol. 102, pp. 9518-9524, 1998. 
[11] J. Hulliger, O. König and R. Hoss, "Polar Inclusion-Compounds of Perhydrotriphenylene (PHTP) and Efficient Nonlinear-Optical Molecules", Adv. Mater., Vol. 7, pp. $719-721,1995$.

[12] J. Hulliger, A. Quintel, M. Wübbenhorst, P. J. Langley, S. Roth, and P. Rechsteiner, "Theory and pyroelectric characterization of polar inclusion compounds of perhydrotriphenylene", Opt. Mater., Vol. 9, pp. 259-264, 1998.

[13] A. Huwe, F. Kremer, P. Behrens and W. Schwieger, "Molecular dynamics in confining space: From the single molecule to the liquid state", Phys. Rev. Lett., Vol. 82, pp. 2338-2341, 1999.

[14] A. Huwe, M. Arndt, F. Kremer, C. Haggenmüller and P. Behrens, "Dielectric investi- gations of the molecular dynamics of propanediol in mesoporous silica materials", J. Chem. Phys., Vol, 107, pp. 9699-9701, 1997.

[15] I. Leike and F. Marlow, "One-dimensional phase transition of dipolar molecules inside zeolite pores", Zeolites, Vol. 16, pp. 65-69, 1996.

This manuscript is based on a paper given at the 10th International Symposium on Electrets, Delphi, Greece, 22-24 September 1999.

Manuscript was received on 14 March 2000, in revised form 16 May 2000. 